\title{
Phylogenetic Group/Subgroups Distributions, Virulence Factors, and Antimicrobial Susceptibility of Escherichia coli Strains from Urinary Tract Infections in Hatay
}

\author{
Ebru Şebnem Yılmaz ${ }^{[1]}$ and Özkan Aslantaş ${ }^{[2]}$ \\ [1]. Department of Biology, Faculty of Art and Science, Hatay Mustafa Kemal University, TR-31060 Hatay, Turkey. \\ [2]. Department of Microbiology, Faculty of Veterinary Medicine, Hatay Mustafa Kemal University, TR-31060 Hatay, Turkey.
}

\begin{abstract}
Introduction: Nosocomial and community acquired urinary tract infections (UTIs) are one of the most encountered infections in the world. Methods: This study aimed to determine the antibiotic susceptibility, phylogeny, and virulence genes of 153 Escherichia coli strains isolated from UTIs. Antimicrobial susceptibility of the isolates to different classes of antimicrobials was determined by the VITEK-2 automated system. Presence of virulence genes and phylogenetic groups were investigated by PCR. Results: Regarding susceptibility to antimicrobials, ampicillin resistance was most abundant (67.3\%), followed by amoxicillin-clavulanic acid (50.9\%); least abundant was resistance to amikacin (1.3\%) and nitrofurantoin (1.3\%). Multi drug resistance (MDR) was observed in 34.6\% of the isolates, and all isolates were found to be susceptible to imipenem, meropenem and fosfomycine. The majority of the isolates belonged to the phylogenetic group B2 (35.9\%), followed by A1 (20.9\%), D1 (18.9\%), D2 (12.4\%), A0 (\%5.9), B1 (3.9\%) and B2 (1.9\%). Among E. coli strains examined, $49 \%$ had iuc $\mathrm{D}, 32.7 \%$ pap $\mathrm{E}-\mathrm{F}, 26.1 \%$ pap $\mathrm{C}, 15 \%$ cnf $2,11.1 \%$ sfa, $7.8 \%$ cnf $1,1.3 \%$ afa $\mathrm{E}, 1.3 \%$ afa $\mathrm{D}, 1.3 \%$ hlyA, $0.7 \%$ f $17 a-\mathrm{A}, 0.7 \% \operatorname{clp} \mathrm{G}$ and $0.7 \%$ eaeA genes. Conclusions: Our research demonstrated that virulence factors were distributed among different phylogroup/subgroups, which play a role in UTIs pathogenesis in humans. For this reason, complex and detailed studies are required to determine the relationship between virulence factors and specific E. coli strains that cause UTIs in humans.
\end{abstract}

Keywords: Urinary Tract Infections. Virulence genes. Phylogenetic group. Antimicrobial resistance.

\section{INTRODUCTION}

Urinary tract infections (UTIs) are one of the most common infections, affecting both outpatients and inpatients worldwide ${ }^{1}$. Uropathogenic Escherichia coli (UPEC), classified as Extraintestinal pathogenic E. coli (ExPEC), are one of the most predominant causes of UTIs ${ }^{2}$. UPEC strains have several virulence factors which play an important role in the pathogenesis of infections. These virulence factors include both structural (fimbriae, pili, curli, flagella) and secreted (toxins, iron-acquisition) systems ${ }^{3}$, are related to colonization and durability of bacteria in the urinary system ${ }^{4}$. In addition, it has been shown that $E$. coli strains causing UTIs have a higher prevalence rate of virulence genes than commensal $E$. coli strains 5 .

\footnotetext{
Corresponding author: Ebru Şebnem Yılmaz.

e-mail: ebrusebnem@gmail.com

(D) 0000-0001-6124-4832

Received 13 September 2019

Accepted 17 December 2019
}

Based on three genetic marker, including chuA, yja A and DNA fragment TSPE4.C2, E. coli strains were mainly divided into four phylogenetic groups (A, B1, B2 and D) by Clermont et al. $(2000)^{6}$. Escobar-Páramo et al. (2004) further divided these phylogenetic groups into subgroups according to presence or absence of the chuA, yjaA genes and the DNA fragment TSPE4.C2 including A0, $\mathrm{A} 1, \mathrm{~B} 1, \mathrm{~B} 2, \mathrm{~B} 2_{3}, \mathrm{D} 1, \mathrm{D}^{7}$. Previous phylogenetic analysis revealed that ExPEC strains causing UTIs mainly belonged to phylogenetic groups $\mathrm{B} 2$ or $\mathrm{D}$, but commensal strains predominantly belonged to phylogenetic groups $\mathrm{B} 1$ or $\mathrm{A}^{4,8}$. Phylogenetic grouping of $E$. coli isolates is of importance not only for understanding of $E$. coli populations, but also elucidating the relationship between strains and disease.

As observed in other bacterial pathogens, increasing antimicrobial resistance in ExPEC strains poses a serious public health threat by decreasing available treatment options for UTIs. Therefore, continuous surveillance of ExPEC strains for antimicrobial susceptibility may provide useful information that will assist physicians in administering effective UTI treatment ${ }^{9}$. 
Previously, there have been a few studies featuring virulence properties, antibiotic resistance, and its relationship with phylogenetic groups among E. coli associated with UTIs in Turkey ${ }^{10-13}$. Therefore, the main objective of this study was to determine the antimicrobial susceptibility, phylogeny, and virulence genes of E. coli isolated from patients admitted to Hatay State Hospital with UTI complaint.

\section{METHODS}

E. coli strains were isolated from urine samples collected from patients admitted to Antakya State Hospital with complaint of UTI between January and June 2014. Isolates were included in the study when a pure culture containing $>10^{5} \mathrm{cfu} / \mathrm{ml}$ was acquired. The isolates were identified with conventional biochemical tests ${ }^{14}$ (Gram staining, oxidase, IMVIC), and confirmed by polymerase chain reaction (PCR) targeting E. coli specific $16 \mathrm{~S}$ rRNA ${ }^{15}$.

Antimicrobial susceptibility of the isolates were performed using an automated method (VITEK ${ }^{\circledR}$ BioMérieux). Susceptibility to 17 antimicrobials including ampicillin, amikacin, amoxycillinclavulanic acid, cefazolin, cefepime, cefoxitin, ceftriaxone, cefuroxime, ciprofloxacin, fosfomycin, gentamicin, imipenem, meropenem, nitrofurantoin, norfloxacin, trimethoprimsulfamethoxazole and piperacillin-tazobactam was tested using a Gram Negative Susceptibility card (AST-N325). The isolates showing resistance to three or more antimicrobials from different classes of antimicrobials were categorized as multi drug resistant (MDR).

Bacterial genomic DNA was acquired by boil extraction method $^{16}$. Phylogenetic grouping of the isolates was determined using multiplex $\mathrm{PCR}^{6}$. The identification of phylogenetic groups and subgroups (A0, A1, B1, B2, B2 ${ }_{3}, \mathrm{D} 1$, D2) were determined based on presence or absence of the $c h u \mathrm{~A}, y j a \mathrm{~A}$ genes and the DNA fragment TspE4-C2 as previously described by Escobar-Páramo et al ${ }^{7}$.

The frequency of virulence genes $(p a p \mathrm{C}$, pap $\mathrm{E}-\mathrm{F}, s f a / f o c \mathrm{DE}$, cnfl, iucD, hlyA, afa D-8, afa E-8, clpG, cnf2, f17A, f17a-A, f17b-A, f17c-A, f17d-A, stx $1, s t x 2$, and eaeA) were investigated using PCR protocols ${ }^{15,17-21}$.

Statistical differences among phylogenetic groups/subgroups, virulence genes, and antimicrobial susceptibility results were determined using Pearson's chi-square test. SPSS 14.01 was used for statistical analysis. In all statistical analyses a level of significance of 0.05 was adopted.

\section{RESULTS}

A total of 153 strains isolated from patient urine specimens were identified as E. coli based on standard biochemical tests and PCR amplification of the targeted 16S rRNA (Figure 1).

Antimicrobial susceptibility testing revealed that all isolates were susceptible to imipenem, meropenem and fosfomycine. Various rates of resistance to ampicillin $(67.3 \%, \mathrm{n}=103)$, amoxicillin-clavulanic acid $(50.9 \% ; n=78)$, cefazolin $(45.1 \%, n=69)$, trimethoprimsulfamethoxazole $(45.1 \%, \mathrm{n}=69)$, cefuroxime $(38.65 \%, \mathrm{n}=59)$, ceftriaxone $(36.6 \%, \mathrm{n}=56)$, ciprofloxacin $(35.9 \%, \mathrm{n}=55)$, cefepime $(35.9 \%, \mathrm{n}=55)$, cefoxitin $(5.2 \%, \mathrm{n}=8)$, norfloxacin $(32.7 \%, \mathrm{n}=50)$, gentamicin $(20.9 \%, \mathrm{n}=36)$, tazobactam-ticarbenicillin $(19.6 \%$;

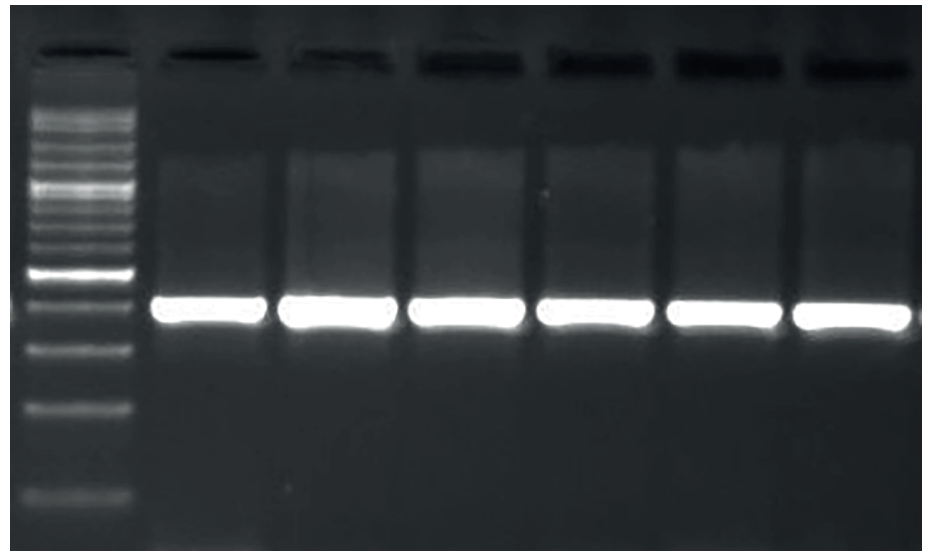

FIGURE 1: PCR amplification of E. coli specific 16S rRNA gene (401 bp).

$\mathrm{n}=30)$, amikacin $(1.3 \%, \mathrm{n}=2)$ and nitrofurantoin $(1.3 \%, \mathrm{n}=2)$ were observed (Figure 2). MDR was observed in $34.6 \%(\mathrm{n}=53)$ of the isolates. Forty $(26.1 \%)$ isolates were found to be susceptible to all antimicrobials tested. There were no statistically significant differences among MDR, non-MDR, and susceptible isolates among phylogenetic groups/subgroups $(\mathrm{P}>0.672)$.

Phylogenetic grouping and subgrouping was determined as follows: 55 (35.9\%) isolates belonged to group $\mathrm{B} 2_{3}, 32$ (20.9\%) belonged to group A1, 29 (18.9\%) belonged to D1, 19 (12.4\%) belonged to $\mathrm{D} 2,9(5.9 \%)$ belonged to A0, $6(3.9 \%)$ belonged to B1, and $3(1.9 \%)$ belonged to B2 (Figure 3).

Of the 153 E. coli isolates, $109(71.2 \%)$ isolates carried at least one virulence gene. Distribution of virulence genes was detected as $i u c \mathrm{D}(49 \%, \mathrm{n}=75)$, pap $\mathrm{E}-\mathrm{F}(32.7 \%, \mathrm{n}=50)$, pap $\mathrm{C}(26.1 \%$, $\mathrm{n}=40)$, chf2 $(15 \%, \mathrm{n}=23), \operatorname{sfa}(11.1 \%, \mathrm{n}=17), \operatorname{cnf} 1(7.8 \%, \mathrm{n}=12)$, afa $\mathrm{E}(1.3 \%, \mathrm{n}=2)$, afaD $(1.3 \%, \mathrm{n}=2)$, hly $\mathrm{A}(1.3 \%, \mathrm{n}=2), f 17 a-\mathrm{A}$ $(0.7 \%, \mathrm{n}=1), \operatorname{clp} \mathrm{G}(0.7 \%, \mathrm{n}=1)$ and eae $\mathrm{A}(0.7 \%, \mathrm{n}=1)$, respectively. In addition, 26 different virulence gene profiles were observed among the isolates. Distribution of virulence gene profiles based on phylogenetic groups/subgroups among the isolates were given in Table 1. Statistically significant differences were observed between the phylogenetic groups and the isolates with and without the virulence gene $(\mathrm{P}<0.001)$.

\section{DISCUSSION}

Determination of antimicrobial resistance and virulence properties of E. coli strains isolated from UTIs are of importance, especially in hospitalized patients, allowing physicians to provide alternative treatment options, reducing the risk of complications, and optimizing ongoing infection control programs ${ }^{9}$. Because UTIs are often treated empirically by physicians, it is therefore necessary to understand the epidemiological data related to agents causing infection in order to improve patient outcomes ${ }^{22}$.

Increased antimicrobial resistance rates, particularly for beta-lactams, sulfamethoxazole-trimethoprim, third generation cephalosporins, and fluoroquinolones, has led to challenges in clinical practice ${ }^{23}$. In this study, nearly half of the isolates were resistant to the majority of the tested antimicrobials, with $34.6 \%$ 


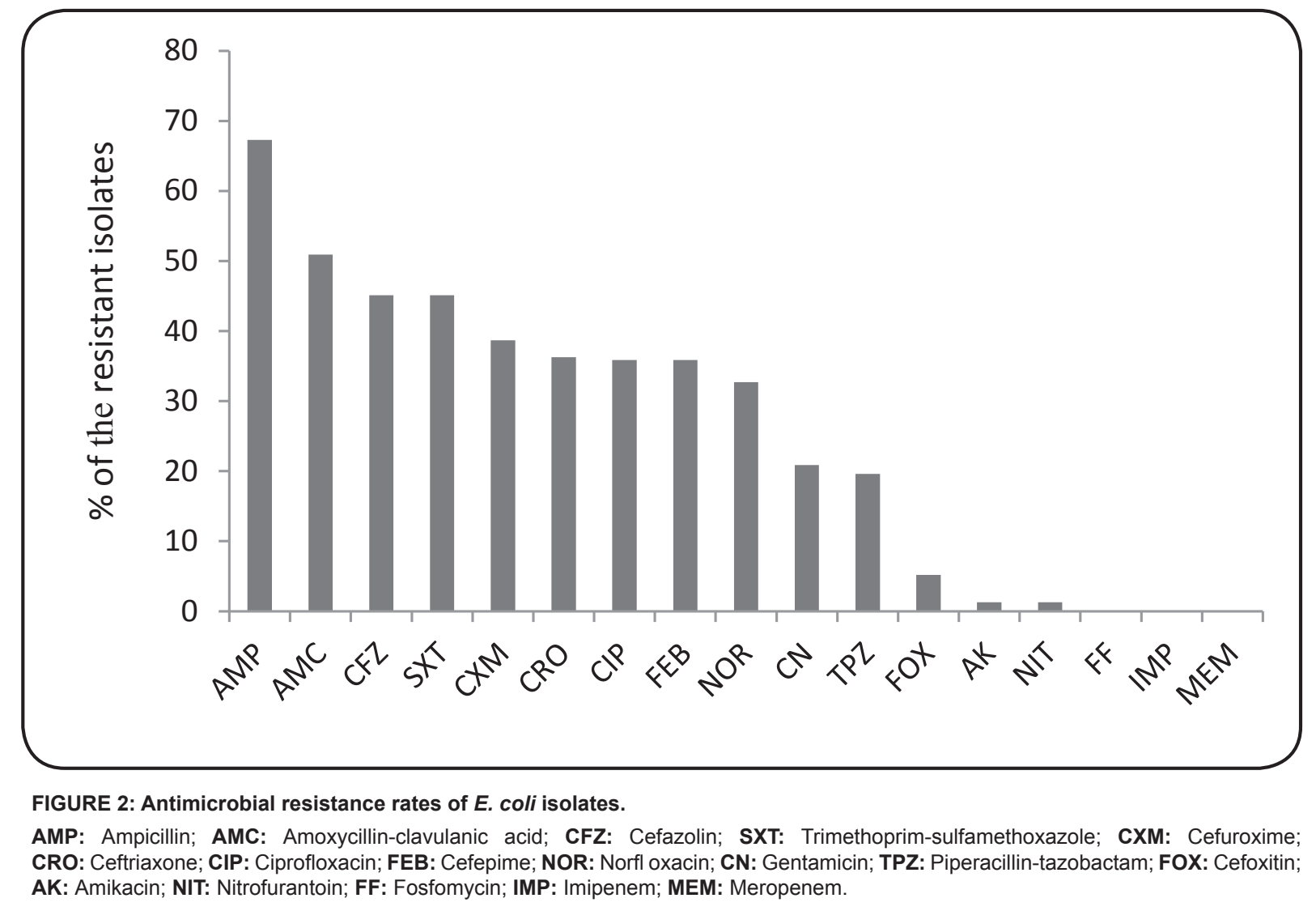

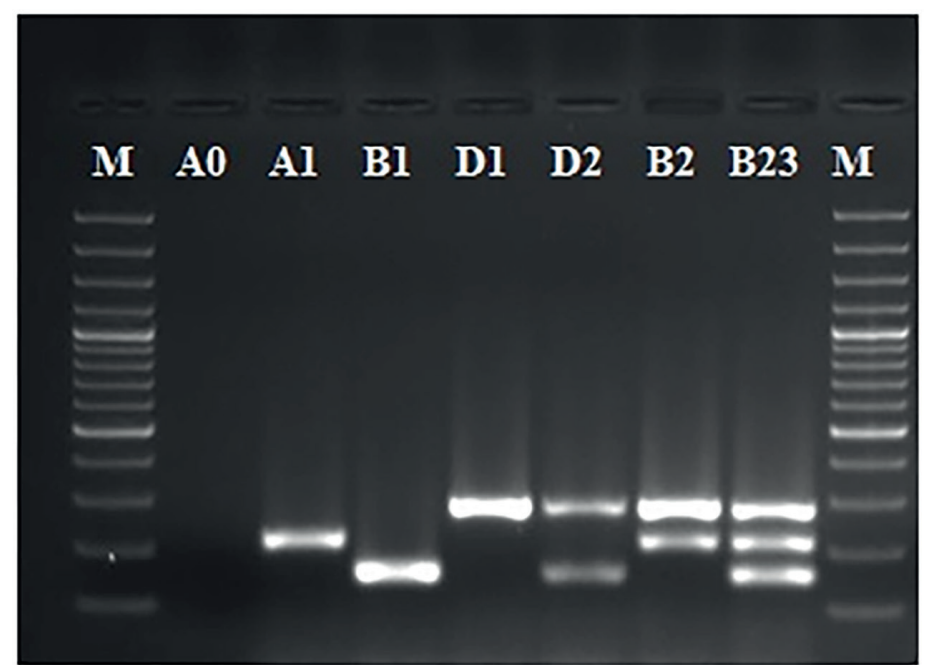

FIGURE 3: Phylogenetic groups determined among E. coli isolates.

of the strains demonstrating MDR, which is in agreement with previous studies conducted in different regions of Turkey ${ }^{12,24}$. In accordance with the results of the study, $67.3 \%, 50.9 \%, 45.1 \%$, $45.1 \%, 38.7 \%, 36.6 \%, 35.9 \%, 35.9 \%$, and $32.7 \%$ were resistant to ampicillin, amoxicillin-clavulanic acid, cefazolin, trimethoprimsulfamethoxazole, cefuroxime, ceftriaxone, ciprofloxacin, cefepime, and norfloxacin, respectively, which are the first-line therapeutic agents used for UTI treatment ${ }^{23,25}$. These resistance rates may be explained by the frequent prescription of these antimicrobials in empirical treatment of UTIs.

UPEC strains have numerous virulence factors that enable bacteria to colonize the urinary tract and overcome various host defense mechanisms ${ }^{26,27}$. In this study, $28.8 \%$ of the isolates were negative for examined genes. On the other hand, $71.2 \%$ of the isolates were positive for at least one of the virulence genes examined. Of these virulence factors, adhesion molecules have an important role in the promotion of colonization, invasion, and replication within uroepithelial cells ${ }^{26}$. In this study, the most prevalent adhesion genes were papE-F $(32.7 \%, \mathrm{n}: 50)$ and pap $\mathrm{C}$ (26.1\%, n: 40), followed by $s f a(11.1 \%, \mathrm{n}: 17)$, afa $\mathrm{E}(1.3 \%, \mathrm{n}: 2)$, and $a f a \mathrm{D}(1.3 \%, \mathrm{n}: 2)$, respectively. Presence of $\mathrm{P}$ fimbria is well documented to be associated with pyelonephritis and cystitis ${ }^{1}$. In a study conducted by Munkhdelger et al. (2017), the frequency of fim $\mathrm{H}$, pap $\mathrm{C}$, pap $\mathrm{GII}$, afa/draBC, sfalfoc $\mathrm{DE}$ and pap $\mathrm{GIII}$ was $89.9 \%$, $20.3 \%, 17.6 \%, 15.5 \%, 8.8 \%$ and $1.4 \%{ }^{28}$. In another study conducted in Brazil, Tiba et al. (2008) reported frequency of the virulence genes $f i m \mathrm{H}$, papC, sfa, and afa to be $97.5 \%, 32.7 \%, 27.8 \%$, and $6.2 \%$, respectively ${ }^{29}$. In Mexico, Paniagua-Contreras et al. (2015) found the prevalence of fim, pap and pap GII as $61.3 \%, 24.7 \%$, and $21.1 \%$, respectively ${ }^{1}$. The $s f a$ gene was found in twelve $(70.6 \%)$ of the isolates together with pap genes. Shetty et al. (2014) explained that co-existence of these two genes are due to their localization on the same pathogenicity island of UPEC strains ${ }^{30}$. In addition, most of the isolates carried multiple adhesion genes, indicating 
TABLE 1: Distribution of virulence gene profiles according to phylogenetic group/subgroups among $E$. coli isolates.

\begin{tabular}{|c|c|c|c|c|c|c|c|}
\hline \multirow{2}{*}{ Virulence Genes } & \multicolumn{7}{|c|}{ Phylogenetic Group/Subgroup } \\
\hline & A0 & A1 & B1 & B2 & $\mathrm{B}_{3}$ & D1 & D2 \\
\hline iucD, papC, cnf1, papE-F, sfa, cnf2 & & & & 1 & 1 & & \\
\hline papC, papE-F, cnf-1, cnf2, sfa & & & & & 4 & & \\
\hline iucD, papC, papE-F, cnf1, cnf2 & & & & 1 & & & \\
\hline$i u c \mathrm{D}, \mathrm{f} 17 \mathrm{a}-\mathrm{A}, \mathrm{afaD}, \mathrm{afaE}$ & & 1 & & & & & \\
\hline papC, papE-F, cnf2, sfa & & & & & 3 & & \\
\hline$i u c \mathrm{D}, \mathrm{cnf}-1, \mathrm{cnf2}, \mathrm{sfa}$ & & & & & 1 & & \\
\hline eaeA, papC, papE-F & & & & & & 1 & \\
\hline iucD, papC, papE-F & & 1 & & & 7 & 10 & 1 \\
\hline iucD, papC, sfa & & & & & 1 & & \\
\hline$i u c \mathrm{D}, \mathrm{afaD}, \mathrm{afaE}$ & 1 & & & & & & \\
\hline$c n f-1, c n f 2, s f a$ & & & & & 3 & & \\
\hline papC, sfa, cnf2 & & & & & 1 & & \\
\hline papC, papE-F & & 1 & & & 2 & 1 & 1 \\
\hline iucD, papE-F & & 3 & & & 1 & 5 & \\
\hline$i u c \mathrm{D}, \mathrm{cnf2}$ & & 1 & & & & & \\
\hline iucD, papC & & & & & 2 & & \\
\hline papE-F, sfa & & & & & 1 & & \\
\hline sfa, cnf2 & & & & & 1 & & \\
\hline$c / p G, h l y A$ & & & & 1 & & & \\
\hline cnf2, hlyA & & & 1 & & & & \\
\hline cnf2 & 1 & 3 & 1 & & & & \\
\hline$i u c D$ & & 7 & 1 & & 24 & 2 & 3 \\
\hline papE-F & & & & & & 3 & 2 \\
\hline$f 17 \mathrm{~A}$ & & 1 & & & & & \\
\hline papC & & & & & & & 1 \\
\hline$c n f-1$ & & & 1 & & & & \\
\hline Negative & 7 & 14 & 2 & & 3 & 7 & 11 \\
\hline Total & 9 & 32 & 6 & 3 & 55 & 29 & 19 \\
\hline
\end{tabular}

that the isolates had the ability to adhere to the urinary tract and subsequently cause infection. It has been reported that ExPEC strains mainly belong to groups B2 and D, and have higher virulence genes in relation with isolates considered to be commensal, which belong to the phylogenetic groups $\mathrm{A}$ and $\mathrm{B} 1^{31,32}$. Similarly, the phylogenetic groups D1 $(29,18.9 \%)$ and B2 $3(55,35.9 \%)$ were the most common among the isolates carrying virulence genes in the study. In a study carried out in Mexico, Miranda-Estrada et al. (2017) reported that the majority of the isolates belonged to group B2 $(60 \%)$ and harbored a high number of virulence factors ${ }^{33}$. A similar result was reported in Pakistan by Bashir et al. (2012), who found $50 \%$ of UPEC isolates belong to group B2, and to a lesser extent, groups A1 and B1 (19\%) $)^{34}$. Lee et al. (2015) also reported high prevalence of virulence factors in groups B2 (79.31\%) and D (15.51\%), followed by groups A (3.44\%) and B1 (1.72\%) in South Korea $^{8}$. On the other hand, in this study, $26.8 \%$ and $3.9 \%$ of the isolates were found to belong to the commensal groups A and D. Our results confirmed this hypothesis not only in ExPEC strains, but also in the commensal E. coli strains that can cause UTIs ${ }^{35,36}$. In addition, Duriez et al. (2001) suggested that the distribution of B1, 
A and D groups in each population can vary according to various factors (geographic/climatic conditions, dietary factors, the use of antibiotics, host genetic factors) and commensal strains can acquire virulence factors and become potentially pathogenic ${ }^{37}$.

In conclusion, various rates of resistance and virulence factors were determined among the isolates. Therefore, monitoring of E. coli isolates should be performed for the effective treatment of UTIs. The results of the study also revealed that $E$. coli isolates from UTIs belong to different phylogroups/subgroups (mainly B2 ), and harbor single or various virulence gene combinations. For this reason, more detailed studies are needed to determine the relationship between virulence traits and certain E. coli clones that cause UTIs in humans.

\section{AUTHORS' CONTRIBUTION}

E.Ş.Y and Ö.A: conceived of the presented idea; E.Ş.Y and Ö.A: designed and performed experiments; E.Ş.Y and Ö.A: verified and checked the analytical methods; Ö.A; performed experimental data analyses. Both of author discussed the results and contributed to the final manuscript.

\section{CONFLICT OF INTEREST}

The authors declare that there is no conflict of interest.

\section{LIMITATION}

This study's limitation is the serogroup assay. Serogroup assays are used for accurate $E$. coli identification and for epidemiological investigations of $E$. coli outbreaks.

\section{ETHICAL DISCLOSURE}

Ethical approval was obtained from the Ethical Committee of Hatay Mustafa Kemal University (Protocol code: 2011/18-14). The authors declare that no experiments were performed on humans or animals for this study.

\section{FINANCIAL SUPPORT}

This study was granted by Hatay Mustafa Kemal University Scientific Research Fund (Project Number: 267).

\section{REFERENCES}

1. Paniagua-Contreras GL, Monroy-Perez E, Rodriguez-Moctezuma JR, Dominguez-Trejo P, Vaca-Paniagua F, Vaca S. Virulence factors, antibiotic resistancephenotypes and O-serogroups of Escherichia coli strains isolated from community acquired urinary tract infection patients in Mexico. J Microbiol Immunol Infect. 2015;50(4):478-85.

2. Yun KW, Kim HK, Park HK, Kim W, Lim IS. Virulence factors of uropathogenic Escherichia coli of urinary tract infections and asymptomatic bacteriuria in children. J Microbiol Immunol Infect. 2014;47(6):455-61.

3. Terlizzi ME, Gribaudo G, Maffei ME. UroPathogenic Escherichia coli (UPEC) infections: virulence factors, bladder responses, antibiotic, and non-antibiotic antimicrobial strategies. Front Microbiol. 2017;8:1566.

4. Giray B, Uçar FB, Aydemir SS. Characterization of uropathogenic Escherichia coli strains obtained from urology outpatient clinic of Ege medical faculty in İzmir. Turk J Med Sci. 2012;42(1):1328-37.

5. Forsyth VS, Armbruster CE, Smith SN, Pirani A, Springman AC, Walters MS, et al. Rapid growth of uropathogenic Escherichia coli during human urinary tract infection. MBio. 2018;9(2):1-13.
6. Clermont O, Bonacorsi S, Bingen E. Rapid and simple determination of the Escherichia coli phylogenetic group. Appl Environ Microbiol. 2000;66(10):4555-8.

7. Escobar-Páramo P, Grenet K, Le Menac'h A, Rode L, Salgado E, Amorin C, et al. Large-scale population structure of human commensal Escherichia coli isolates. Appl Environ Microbiol. 2004;70(9):5698-700.

8. Lee JH, Subhadra B, Son YJ, Kim DH, Park HS, Kim JM, et al. Phylogenetic group distributions, virulence factors andantimicrobial resistance properties of uropathogenic Escherichia coli strains isolated from patients with urinary tract infections in South Korea. Lett Appl Microbiol. 2015;62(1):84-90.

9. Aghemwenhio IS, Timilehin AA, Alpheus GA. Susceptibility of beta-haemolytic Escherichia coli to commonly used antibiotics in selected hospitals in Delta State, Southern Nigeria. Arc Clin Microbiol. 2017;8(2):36.

10. Giray B, Ucar F, Aydemir S. Genotypic analysis of Escherichia coli strains that cause urosepsis in the Aegean region. Turk J Med Sci. 2016;46(5):1518-27.

11. Bozcal E, Eldem V, Aydemir S, Skurnik M. The relationship between phylogenetic classification, virulence and antibiotic resistance of extraintestinal pathogenic Escherichia coli in İzmir province, Turkey. Peer J. 2018;6:e5470.

12. Paykoc EI, Turkyilmaz S. Investigation of $\mathrm{P}$ fimbriae presence in Escherichia coli strains isolated from urine samples in human and their antibacterial resistance. Jundishapur J Microbiol. 2018;11(9):e66119.

13. Düzgün AÖ, Okumuş F, Saral A, Çiçek AÇ, Cinemre S. Determination of antibiotic resistance genes and virulence factors in Escherichia coli isolated from Turkish patients with urinary tract infection. Rev Soc Bras Med Trop. 2019;52:e20180499.

14. Procop G, Church D, Hall G, Janda W, Koneman E, Schrekenberger P, et al. Koneman's color atlas and textbook of diagnostic microbiology.7th ed. Lippincott Williams and Wilkins: Philadelphia; 2017. 360-6 p.

15. Wang G, Clark CG, Rodgers FG. Detection in Escherichia coli of the genes encoding the major virulence factors, the genes defining the O157:H7 serotype, and components of the type 2 Shiga toxin family by multiplex PCR. J of Clinical Microbiology. 2002;40(10):3613-19.

16. Ahmed AM, Motoi Y, Sato M, Maruyama A, Watanabe H, Fukumoto $\mathrm{Y}$, et al. Zoo animals as reservoirs of gram-negative bacteria harboring integrons and antimicrobial resistance genes. Appl Environ Microbiol. 2007;73(20):6686-90.

17. Yamamoto S, Tsukamoto T, Terai A, Kurazono H, Takeda Y, Yoshida O. Distrubiton of virulence factors in E. coli isolated from urine of cystitis patients. Microbiol Immunol. 1995; 39(6):401-4.

18. Bertin Y, Martin C, Oswald E, Girardeau JP. Rapid and specific detection of F17-related pili and adhesin genes in diarrheic and septicemic Escherichia coli strains by multiplex PCR. J Clin Microbiol. 1996;34(12):2921-8.

19. Bertin Y, Martin C, Girardeau JP, Pohl P, Contrepois M. Association of genes encoding P fimbriae, CS31A antigen and EAST 1 toxin among CNF1-producing Escherichia coli strains from cattle with septicemia and diarrhea. FEMS Microbiol Lett. 1998;162(2):235-9.

20. Lalioui L, Jouve M, Gounon P, Le Bouguenec C. Molecular cloning and characterization of the afa 7 and afa 8 gene clusters encoding afimbrial adhesins in Escherichia coli strains associated with diarrhea or septicemia in calves. Infect Immun. 1999;67(10):5048-59.

21. Kaipainen T, Pohjanvirta T, Shpigel NY, Shwimmer A, Pyörälä S, Pelkonen S. Virulence factors of Escherichia coli isolated from bovine clinical mastitis. Vet Microbiol. 2002;85(1):37-46. 
22. Flores-Mireles AL, Walker JN, Caparon M, Hultgren SJ. Urinary tract infections: epidemiology, mechanisms of infection and treatment options. Nat Rev Microbiol. 2015;13(5):269-284.

23. Chiu CC, Lin TC, Wu RX, Yang YS, Hsiao PJ, Lee Y, et al. Etiologies of community-onset urinary tract infections requiring hospitalization and antimicrobial susceptibilities of causative microorganisms. J Microbiol Immunol Infect. 2017;50(6):879-85.

24. Yılmaz N, Ağuş N, Bayram A, Şamlıŏlu P, Şirin MC, Derici YK and Hanc1 SY. Antimicrobial susceptibilities of Escherichia coli isolates as agents of community-acquired urinary tract infection (2008-2014). Turk J Urol. 2016;42(1):32-6.

25. Stultz JS, Doern CD, Godbout E. Antibiotic resistance in pediatric urinary tract infections. Curr Infect Dis Rep. 2016;18(12):40.

26. Bien J, Sokolova O, Bozko P. Role of uropathogenic Escherichia coli virulence factors in development of urinary tract infection and kidney damage. Int J Nephrol. 2012;2012:681473.

27. Ko"ves B and Wullt B. The roles of the host and the pathogens in urinary tract Infections. Eur Urol Suppl. 2016;15(4):88-94.

28. Munkhdelger Y, Gunregjav N, Dorjpurev A, Juniichiro N, Sarantuya J. Detection of virulence genes, phylogenetic group and antibiotic resistance of uropathogenic Escherichia coli in Mongolia. J Infect Dev Ctries. 2017;11(1):51-7.

29. Tiba MR, Yano T, Leite Dda S. Genotypic characterization of virulence factors in Escherichia coli strains from patients with cystitis. Rev Inst Med Trop Sao Paulo. 2008;50(5): 255-60.

30. Shetty AV, Kumar SH, Shekar M, Shetty AK, Karunasagar I, Karunasagar I. Prevalence of adhesive genes among uropathogenic Escherichia coli strains isolated from patients with urinary tract infection in Mangalore. Indian J Med Microbiol. 2014;32(2):175-8.
31. Chapman TA, Wu X, Barchia I, Bettelheim KA, Driesen S, Trott D, et al. Comparison of virulence gene profiles of $E$. coli strains isolated from healthy and diarrheic swine. Appl Environ Microbiol. 2006;72(7):4782-95.

32. Chakraborty A, Saralaya V, Adhikari P, Shenoy S, Baliga S, Hegde A. Characterization of Escherichia coli phylogenetic groups associated with extraintestinal infections in South Indian population. Ann Med Health Sci Res. 2015;5(4):241-6.

33. Miranda-Estrada LI, Ruíz-Rosas M, Molina-López J, Parra-Rojas I, González-Villalobos E, Castro-Alarcón N. Relationship between virulence factors, resistance to antibiotics and phylogenetic groups of uropathogenic Escherichia coli in two locations in Mexico. Enferm Infecc Microbiol Clin. 2017;35(7):426-33.

34. Bashir S, Haque A, Sarwar Y, Ali A, Anwar MI. Virulence profile of different phylogenetic groups of locally isolated community acquired uropathogenic E. coli from Faisalabad region of Pakistan. Ann Clin Microbiol Antimicrob. 2012;11:23.

35. Moreno E, Andreu A, Pérez T, Sabaté M, Johnson JR, Prats G Relationship between Escherichia coli strains causing urinary tract infection in women and the dominant faecal flora of the same hosts. Epidemiol Infect. 2006;134(5):1015-23.

36. López-Banda DA, Carrillo-Casas EM, Leyva-Leyva M, OrozcoHoyuela G, Manjarrez-Hernández ÁH, Arroyo-Escalante S, et al. Identification of virulence factors genes in Escherichia coli isolates from women with urinary tract infection in Mexico. Biomed Res Int. 2014; 2014:959206.

37. Duriez P, Clermont O, Bonacorsi S, Bingen E, Chaventré A, Elion J, et al. Commensal Escherichia coli isolates are phylogenetically distributed among geographically distinct human populations. Microbiology. 2001;147(Pt 6):1671-6. 